\title{
Three cases of oral syphilis - an overview
}

\author{
L. Jones, ${ }^{1}$ E. L. C. Ong, ${ }^{2}$ A. Okpokam, ${ }^{3}$ P. Sloan ${ }_{1}{ }^{4}$ I Macleod ${ }^{5}$ \\ and K. S. Staines ${ }^{6}$
}

IN BRIEF

- Highlights the dramatic increase in the number of new syphilis cases in the UK.

- Proposes that the first presentation of syphilis may be within the oral cavity.

- Stresses how the oral presentation of syphilis varies through the stages of the disease.

- Looks at diagnosis and curative treatment once syphilis has been suspected.

- Warns how a missed syphilis diagnosis may result in the spread of the disease.

Syphilis is an infectious disease caused by the organism Treponema pallidum. There has been a dramatic increase in the number of new cases of syphilis in the UK over the past decade. Intra-oral ulceration is often the only presenting feature of the disease, which then enters a latent period. A missed diagnosis can often lead to serious complications and may result in further spread of the disease. Three cases are discussed in this paper with varying clinical presentations of the disease. Such a significant increase of syphilis and its high infectivity require the dental profession to increase their awareness of sexually infectious diseases and the appropriate dental management.

\section{INTRODUCTION}

In the UK there was a 951\% increase in the number of new cases of primary or secondary syphilis from 1999 to 2008. (HPA Data, Fig. 1) This increase is accounted for by the male population as the number of new cases in females has stayed fairly consistent over this period. ${ }^{1}$

A significant resurgence of syphilis has also been reported in Europe, North America, Russia and China. Concomitant infection with syphilis and HIV has increased, with significant epidemiological and clinical implications. ${ }^{2}$

Possible reasons for this increase in prevalence are a false sense of security that today sexually transmitted infections are curable, resulting in an increase in sexual promiscuity and alcohol abuse, and a decrease in the use of barrier protection and lack of pertinent knowledge. ${ }^{3}$

${ }^{1 *}$ House Officer, Newcastle Dental Hospital, Richardson Road, Newcastle Upon Tyne, NE2 4AZ; ${ }^{2}$ Consultant Physician and Honorary Senior Lecturer, Department of Infection and Tropical Medicine, Royal Victoria Infirmary, Newcastle upon Tyne, NE1 4LP; ${ }^{3}$ Consultant Pathologist, Newcastle upon Tyne Hospitals NHS Foundation Trust, NE7 7DN; ${ }^{4}$ Professor in the School of Dental Sciences, Newcastle University, Framlington Place, Newcastle upon Tyne, NE2 4HH; ${ }^{5}$ Consultant/ Honorary Clinical Senior Lecturer, DMF Radiology, Newcastle Dental Hospital, Richardson Road, Newcastle Upon Tyne, NE2 4AZ; ${ }^{6}$ Consultant in Oral Medicine, Newcastle Upon Tyne Hospitals NHS Trust, NE7 7DN

${ }^{*}$ Correspondence to: Miss Laura Jones

Email: laura.jones23@nhs.net

\section{Refereed Paper}

Accepted 1 March 2012

DOI: $10.1038 /$ sj.bdj.2012.420

${ }^{\circ}$ British Dental Journal 2012; 212: 477-480

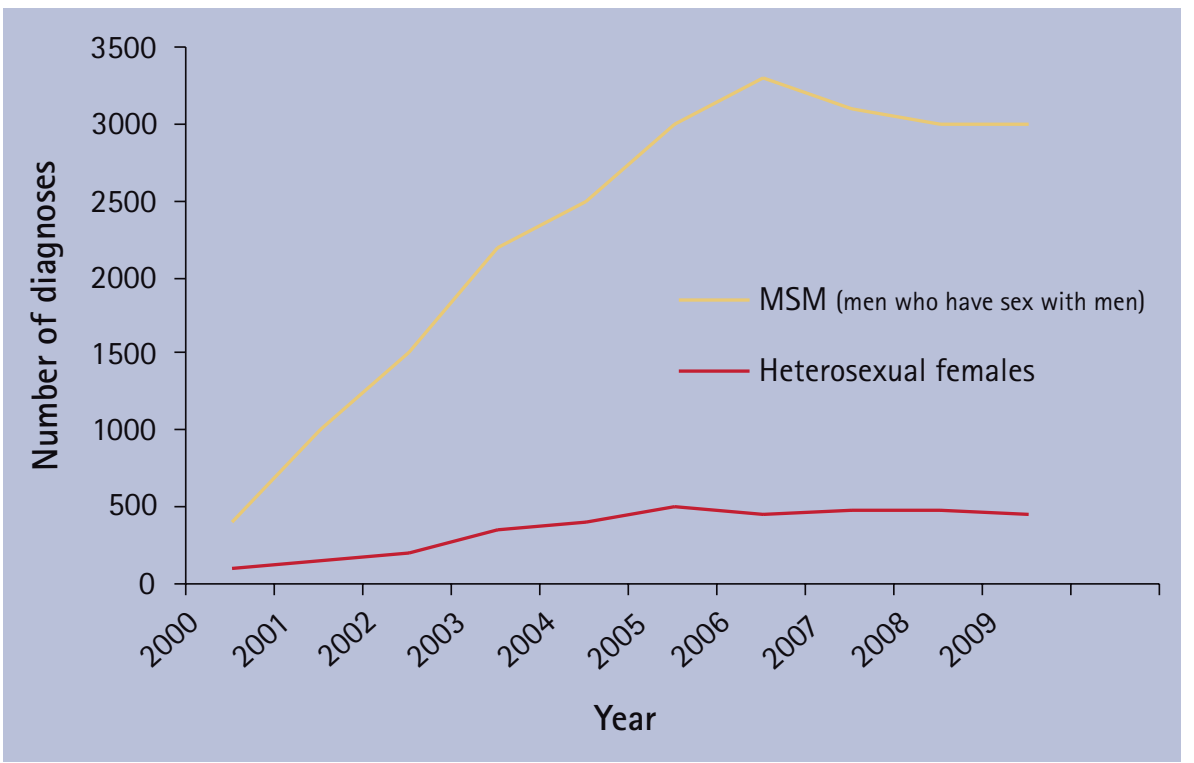

Fig. 1 The increase in the number of diagnosed cases of syphilis in the UK from 2000-2009 extracted from HPA data

Patients may present to a variety of clinicians due to the diverse clinical appearance of both early and late syphilis. Until recently there has been little clinical experience of syphilis among the medical profession. ${ }^{4}$ A major risk group for syphilis infection are homosexual men, as a large number of cases are asymptomatic. Regular serological testing has been recommended for this group. ${ }^{5}$

The only presenting feature in many patients may be the presence of an intraoral lesion. Prompt recognition is necessary to ensure a diagnosis to control further consequences due to unknown infectivity. Syphilis can be transmitted through blood, saliva and direct contact with lesions and is known to be associated with an increased risk of HIV transmission, demonstrating the need for adequate cross infection control in dentistry. ${ }^{6,7}$ We report three cases which initially presented with oral manifestations.

\section{CASE ONE}

A 41-year-old male was referred from a maxillofacial outpatient clinic to the oral medicine department at Newcastle Dental Hospital regarding an ulcer on the dorsum of his tongue. The ulcer had developed 
suddenly three months before with no identifiable precipitating factors and was associated with a constant deep throbbing pain. An initial period of pyrexia and night sweats, which resolved spontaneously after one week, was reported. Medically the patient was fit and well, though taking steroid eye drops for an eye injury. Socially he was single, homosexual, a non-smoker and reported unprotected sexual activity approximately two months before the ulcer developed.

Extra-oral clinical examination was unremarkable. Intra-oral examination revealed a raised ulcer approximately $1 \mathrm{~cm}$ in diameter surrounded by a wall of indurated fibrous tissue (Fig. 2).

A provisional diagnosis of a primary syphilitic chancre was made. An incisional biopsy had been taken in the maxillofacial clinic immediately before referral to our department. The histopathology had been reported as showing hyperplastic squamous epithelium with a subepithelial dense chronic inflammatory reaction infiltrated with plasma cells, with no underlying dysplasia or malignancy. The tissue blocks were retrieved and antibody stains for T. pallidum were performed. Immunohistochemistry confirmed the presence of $T$. pallidum, in a distribution consistent with a primary chancre.

Treponemal antibody test was positive, syphilis IgM was positive, venereal diseases reference laboratory test (VDRL) was 1 in 128 and serodia was $>1,280$. A diagnosis of primary syphilis was made. Treatment consisted of a single dose of intramuscular benzathine penicillin G 2.4 million units (MU). A course of lidocaine lollipops was prescribed to try to alleviate his pain. The patient was referred to a GUM clinic where contact tracing, full sexual screening and counselling regarding safe sexual activity were carried out. The patient reported a history of bisexual activity.

On review in the oral medicine department one week following treatment, the ulcer had reduced in size and at two week review had completely resolved.

\section{CASE TWO}

A 41-year-old woman was also referred to the oral medicine department regarding an asymptomatic white patch on the lateral border of the tongue (Fig. 3). She reported a previous history of

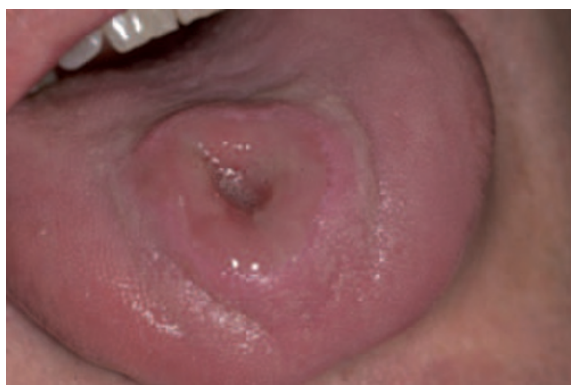

Fig. 2 The primary chancre seen in case one at initial presentation. (Reprinted from the New England Journal of Medicine with permission. Copyright ownership: Staines $K_{t}$ Sloan P. Syphilitic chancre of the tongue. N Engl J Med 2011; 365: e11)

cervical lymphadenopathy and a sore throat approximately two years ago with no other systemic symptoms.

Clinical examination confirmed the presence of white patches on the lateral aspect of the tongue. Biopsy and histopathological examination indicated features consistent with chronic hyperplastic candidosis, but no candidal hyphae were observed and the lesion did not respond to systemic fluconazole. Other possible differential diagnoses from a histopathological perspective were geographic tongue, oral psoriasis, Reiter's syndrome, and finally syphilis. However the clinical features were not consistent with geographic tongue or Reiter's syndrome and there was no history of psoriasis. The T. pallidum screen was carried out to exclude syphilis with consent from the patient.

An HIV test was negative but syphilis serology was positive. Screening was carried out with the treponemal test VDRL, which was reactive at $>1: 32$. This was then confirmed with a treponemal TPPA test, which was reactive at $>1: 1280$. An IgM ELISA was carried out and this was negative. Following these results the patient was referred to the infectious diseases department where a diagnosis of early latent syphilis was confirmed. She received treatment in the form of benzathine penicillin 2.4 MU and intramuscular injections on a weekly basis for three weeks with immediate resolution of the white patches. Contact tracing was performed and the patient's husband was found to be the source of infectivity.

\section{CASE THREE}

A 78-year-old lady was seen in Newcastle Dental Hospital in the mid 1980s by one of the authors (IM) with a carcinoma of her right upper lip. On examination it was

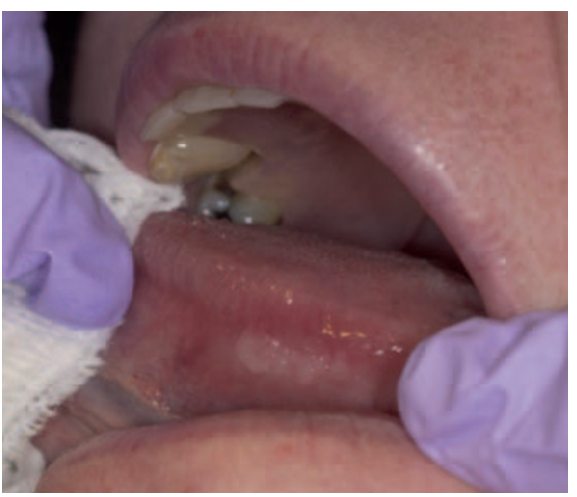

Fig. 3 The presenting lesion in case two: a non-specific well demarcated white patch on the lateral border of the tongue

noted that she had two round perforations in her palate (Fig. 4) and a diffuse keratosis of her tongue (Fig. 5).

These appearances were suggestive of changes seen in tertiary syphilis. A VDRL test was undertaken and confirmed the clinical suspicion. Interestingly, the patient did recollect developing symptoms suggestive of a venereal disease sometime during the Second World War when Newcastle upon Tyne was a major port. Instead of seeking orthodox treatment she attended a well known local chemist shop that treated 'venereal diseases.' The within house remedies which were later found to be based on arsenic, were a likely contributing factor in her cancer development. The patient was lost to follow-up.

\section{DISCUSSION}

\section{Clinical presentation of syphilis: extra-oral}

The range of extra-oral manifestations of syphilis may be classified according to the stage of the disease. There are numerous less common extra-oral manifestations outside the scope of this article. An example of the latter that may be relevant to dentists is the development of cranial nerve palsies in tertiary syphilis. The symptoms of syphilis evolve through four stages: primary, secondary, latent and tertiary. The risk of infectivity and clinical features are closely linked to the stage of the disease. ${ }^{2,3,8}$

\section{Clinical presentation of syphilis: intra-oral}

The oral presentation of syphilis varies through the stages mentioned previously (Table 1). The first intra-oral lesion seen is the primary chancre which typically 


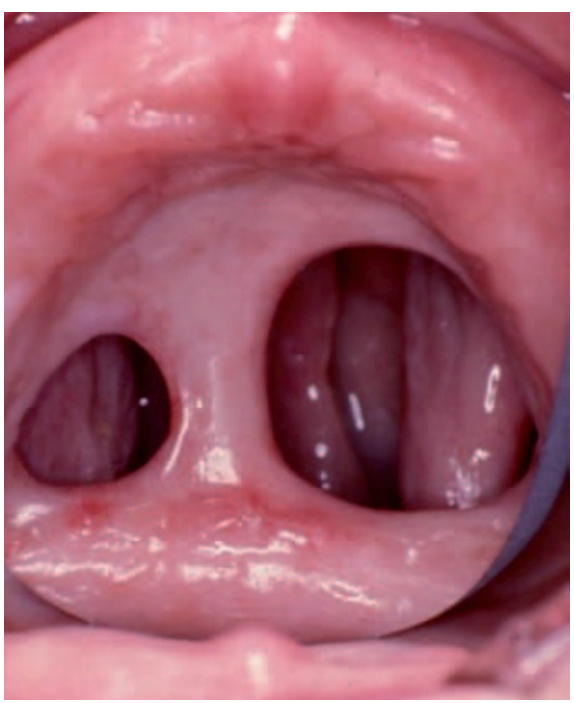

Fig. 4 Palate showing two roughly circular, punched out lesions characteristic of the destruction caused by syphilitic gummas

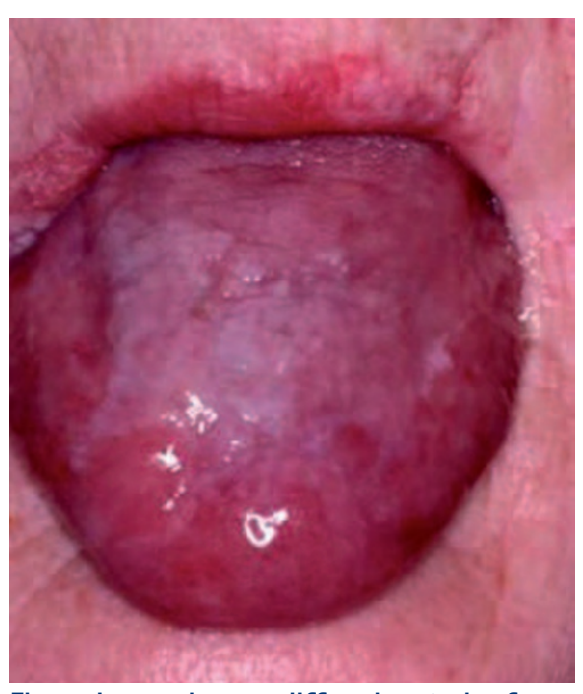

Fig. 5 Image shows a diffuse keratosis of the dorsum of the tongue on a generalised atrophic background, appearances are typical of a syphilitic glossitis. The lip carcinoma with which the patient presented can just be seen on the upper right vermilion border of the lip

affects the tongue, palate, gingiva or lips and has a spontaneous resolution. ${ }^{3}$ The secondary stage of the disease is characterised by snail track ulceration. Lues maligna also known as malignant syphilis is a rare manifestation of secondary syphilis which can involve the oral mucosa. Multiple irregularly distributed mucosal and gingival lesions may develop simultaneously with the classic oral lesions of syphilis described above.

Following this, latent syphilis often does not show any clinical manifestations but occasionally non-specific white patches, 'mucocutaneous relapse', can occur. The final phase of the infection is

\begin{tabular}{|c|c|}
\hline Stage of infection & Intraoral manifestations \\
\hline Primary syphilis & $\begin{array}{l}\text { A chancre involving sites such as tongue, gingivae or lips; regional lymphadenopathy may } \\
\text { be observed. }{ }^{3}\end{array}$ \\
\hline Secondary syphilis & $\begin{array}{l}\text { Raised lesions with a pseudomembrane surrounded by erythema (mucous patches) and/or } \\
\text { snail track oral mucosal ulceration and regional lymphadenopathy. }\end{array}$ \\
\hline Latent syphilis & $\begin{array}{l}\text { Often has no clinical manifestations. However, so-called mucocutaneous relapse with } \\
\text { nonspecific oral mucosal white patches may occur. }{ }^{3}\end{array}$ \\
\hline Tertiary syphilis & $\begin{array}{l}\text { Gumma, which is a crater type ulcer that may involve bone, typically the hard palate. }{ }^{4} \\
\text { White patches may develop intra-orally (syphilitic leukoplakia), which have been reported } \\
\text { as potentially malignant lesions. }\end{array}$ \\
\hline
\end{tabular}

\section{Table 2 Laboratory investigations}

\begin{tabular}{|c|c|c|c|}
\hline \multicolumn{2}{|l|}{ Non-treponemal tests } & \multicolumn{2}{|l|}{ Treponemal tests } \\
\hline $\begin{array}{l}\text { Venereal Diseases Research } \\
\text { Laboratory (VDRL) }\end{array}$ & Liposomes in suspension & FTA-ABS & $\begin{array}{l}\text { Intact treponemes fixed } \\
\text { onto microscopic slides }\end{array}$ \\
\hline Rapid plasma reagin (RPR) & Liposomes in suspension & TPPA (serodia) & $\begin{array}{l}\text { Purified and sonicated } \\
\text { treponemes attached to } \\
\text { gelatin particles. }\end{array}$ \\
\hline $\begin{array}{l}\text { Enzyme immunoassay } \\
\text { (EIA) (Reagin) }\end{array}$ & $\begin{array}{l}\text { VDRL antigen coated } \\
\text { onto microtitre plates }\end{array}$ & \multirow[t]{2}{*}{ Recombinant EIA } & \multirow[t]{2}{*}{$\begin{array}{l}\text { Recombinant antigens } \\
\text { attached to microtitre plates }\end{array}$} \\
\hline $\begin{array}{l}\text { Solid phase erythrocyte } \\
\text { adherence (SPEA) }\end{array}$ & Red blood cells & & \\
\hline
\end{tabular}

\begin{tabular}{|c|c|}
\hline Stage of disease & Treatment \\
\hline Primary syphilis & Benzathine penicillin G 2.4 MU intramuscularly in single dose \\
\hline Secondary syphilis & Benzathine penicillin G 2.4 MU intramuscularly in single dose \\
\hline Latent syphilis of less than one year & Benzathine penicillin G 2.4 MU intramuscularly in single dose \\
\hline Latent syphilis (longer than one year) & $\begin{array}{l}\text { Benzathine penicillin G } 2.4 \text { MU intramuscularly once a week } \\
\text { for three weeks. }\end{array}$ \\
\hline $\begin{array}{l}\text { Tertiary (gummatous - after } 15 \text { years or } \\
\text { cardiovascular syphilis - after } 10-30 \text { years) }\end{array}$ & $\begin{array}{l}\text { Benzathine penicillin G } 2.4 \text { MU intramuscularly once a week } \\
\text { for three weeks. }\end{array}$ \\
\hline Tertiary (neurosyphilis) & $\begin{array}{l}\text { Daily intramuscular procaine penicillin G1.8 - 2.4 MU } \\
\text { and oral probenecid } 500 \mathrm{mg} \text { four times per day, both for } \\
17 \text { days. }{ }^{11}\end{array}$ \\
\hline
\end{tabular}

represented by punched out, crater like ulcers known as gummas, which commonly affect the hard palate region but may also be seen on the tongue. White patches may develop intra-orally (syphilitic leukoplakia) as seen in case three. Syphilitic leukoplakia has been reported as a potentially malignant lesion due to recognition that such lesions may transform. ${ }^{2}$ In case three this complication did arise; however, we suspect that the arsenic treatment may have been a significant aetiological factor.

It is crucial that the correct diagnosis is made, as although the features of the primary chancre often resolve spontaneously within 3-6 weeks, blood borne dissemination occurs leaving behind an

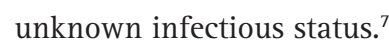

These cases illustrate some of the varying presentations of syphilis to the dental practitioner and also the difficulties in detection and diagnosis. Case one was an atypical presentation of primary syphilis as the major feature of the lesion was acute pain. The syphilitic chancre usually presents as an indurated, painless ulcer. ${ }^{3}$ Case two showed more typical presentations of early latent syphilis in the form of a white patch and case three mucocutaneous 
relapse and the gummatous necrosis seen in tertiary syphilis.

\section{DIAGNOSIS/LABORATORY INVESTIGATIONS}

Syphilis is diagnosed by correlation of clinical findings elicited by a detailed history and relevant clinical examination with investigations such as dark field microscopy, histopathological examination and serology. ${ }^{8}$ Darkfield microscopy is limited to the diagnosis of primary syphilis and is of limited diagnostic value in lesions involving the oral cavity. T. pallidum cannot survive outside the human body, therefore cannot be cultivated for in vitro testing. Histopathology may be helpful and even diagnostic if treponemal organisms can be demonstrated by silver stain or immunostaining. ${ }^{9}$

There are two main categories into which the serological tests for syphilis fall. Those which detect the non-treponemal antibody, known as non-treponemal tests and those that detect the specific treponemal antibody, treponemal tests (Table 2).

Non-treponemal test screening alone is not sufficient for diagnosis due to the potential for false negative results. Appropriate screening is by a treponemal EIA or a combination of a non-treponemal and a treponemal test. ${ }^{7}$ Confirmatory testing of reactive specimens is then required, using a treponemal test of equal sensitivity and greater specificity. Confirmed reactive specimens should be sent to a public health laboratory so that data can be collected and reference testing carried out. The results should be confirmed by testing of a second specimen and referral to a consultant in genitourinary medicine should be made if thought appropriate. Assessment of the stage of infection and monitoring of the efficacy of treatment using a specific treponemal IgM test or a quantitative nontreponemal test is indicated. ${ }^{9}$ This protocol was followed in both current cases and a combination of treponemal and nontreponemal tests were carried out to confirm the diagnosis. Both patients were then referred to a consultant in infectious diseases for further management.

\section{COMPLICATIONS OF SYPHILIS}

Significant complications of syphilis typically, but not exclusively, arise in tertiary syphilis. Neurological complications may range from stroke to dementia. Cardiovascular complications may range from heart valve damage to aneurysm formation. Gummata may develop, typically involving the hard palate and lead to bone destruction. During pregnancy, an infected mother may transmit syphilis to the fetus, which increases the risk of miscarriage or stillbirth. An infected newborn (congenital syphilis) may be associated with significant complications. Also patients with primary syphilis are at an increased risk of acquiring other sexually transmitted diseases such as HIV. ${ }^{6}$

Although an association between syphilis and carcinoma of the tongue has previously been recognised it remains a controversial issue. It has been suggested that the more probable risk factor for malignant transformation of syphilitic leukoplakia is the carcinogenic agents previously used to treat syphilis as described in case three. ${ }^{10}$

\section{MANAGEMENT}

Once syphilis has been suspected, diagnosis and curative treatment are usually straightforward. Treatment is often in the form of intramuscular benzathine penicillin as demonstrated in all three cases (Table 3). Desensitising is recommended for patients with a penicillin allergy. Follow-up with laboratory testing is required to assess treatment efficacy. ${ }^{11}$ The current cases described previously were all treated with intramuscular benzathine penicillin and followed up using a treponemal test. Case three had no documented follow-up. There have been problems reported regarding antimicrobial treatment failure and relapse in HIV positive patients. Second line treatment with oral doxycycline/oral amoxicillin plus probenecid is not fully treponecidal in CSF and therefore suboptimal in HIV positive patients. ${ }^{12}$

These cases demonstrate the varying presentations and management of syphilis. Thorough examination of the oral mucosa and palpation of neck nodes are an important part of the clinical assessment. If suspicion of syphilis as a differential diagnosis is raised then a swift appropriate referral to secondary care is essential. Management will then often require a multidisciplinary approach involving oral and maxillofacial units, oral medicine, genitourinary medicine and infectious diseases as demonstrated in the above cases.

1. Health Protection Agency Syphilis and Lymphogranuloma Venereum: Resurgent Sexually Transmitted Infections in the UK: 2009 report.

2. Kent M E, Romanelli F. Re-examining syphilis: an update on epidemiology, clinical manifestations, and management. Ann Pharmacother 2008: 42: 226-236.

3. Ficarra G, Carlos R. Syphilis: the renaissance of an old disease with oral implications. Head Neck Pathol 2009; 3: 195-206.

4. French P. Syphilis. Br Med J 2007; 334: 143-147.

5. Azariah $S$. An audit of patients treated for syphilis at Auckland Sexual Health Service. NZ Med J 2010; 123: 55-64.

6. Lynn W A, Lightman S. Syphilis and HIV: a dangerous combination. Lancet Infect Dis 2004; 4: 456-466.

7. Little J W. Syphilis: an update. Oral Surg Oral Med Oral Pathol Oral Radiol Endod 2005; 100: 3-9.

8. Hook E W 3rd, Marra C M. Acquired syphilis in adults. New Eng J Med 1992; 326: 1060-1069.

9. Egglestone S I, Turner A J. Serological diagnosis of syphilis. PHLS Syphilis Serology Working Group. Commun Dis Public Health 2000; 3: 158-162.

10. Dickenson A J, Currie W J, Avery B S. Screening for syphilis in patients with carcinoma of the tongue. Br J Oral Maxillofac Surg 1995; 33: 319-320.

11. Kingston $M$, French $P$, Goh B et al. UK national guidelines on the management of syphilis 2008. Int J STD AIDS 2008; 19: 729-740.

12. Nandwani R, Fisher M, Medical Society for the Study of Venereal Diseases HIV Special Interest Group. Clinical standards for the screening and management of acquired syphilis in HIV-positive adults. Int J STD AIDS 2006; 17: 588-593. 